\title{
Investigating the Optimal Recording Duration for Summarising Spatiotemporal Behaviours of Long Lifespan Rotors Using Phase Mapping of Non-Contact Electrograms During Persistent Atrial Fibrillation
}

\author{
Mahmoud Ehnesh ${ }^{1}$, Xin Li $^{1,2,3}$, Nawshin Dastagir ${ }^{4}$, Saaima Ahmad ${ }^{1}$, Taher A Biala ${ }^{1}$, P J Stafford ${ }^{2,3}$, \\ G. André $\mathrm{Ng}^{2,3}$, Fernando S. Schlindwein ${ }^{1,3}$ \\ ${ }^{1}$ Department of Engineering, University of Leicester, Leicester, UK \\ ${ }^{2}$ Department of Cardiovascular Sciences, Glenfield Hospital, Leicester, UK \\ ${ }^{3}$ National Institute for Health Research Leicester Cardiovascular Biomedical Research Centre, \\ Leicester, UK \\ ${ }^{4}$ Auckland Bioengineering Institute, University of Auckland, New Zealand
}

\begin{abstract}
Understanding the spatiotemporal behaviour of 'rotors' in human atrial fibrillation $(A F)$ is important for using them as targets for ablation. This study aims to track the spatiotemporal stability of rotors over $5 \mathrm{~min}$ time interval during persistent atrial fibrillation (PersAF). This study involved 10 PersAF patients, who underwent catheter ablation. 2048 non-contact virtual unipolar electrograms (VEGMs) were simultaneously collected and resampled at $512 \mathrm{~Hz}$, QRST interval removed and reconstructed using a sinusoidal wavelet fitting approach (Kuklik et al. Subsequent density maps of rotors were generated. The VEGM were divided into a total of 60 segments of different durations starting from $5 s, 10 s, 15 s$ and so on. The segments were further divided into; group $A \leq 30 \mathrm{~s}$, group $B>30$, density maps of different time durations were compared with the full 300 $s$. Rotor density maps in segments recorded in group $A$ differed significantly from group B, (CORR: group A $10 \mathrm{~s}$ $=0.47 \pm 0.064$ Vs. $30 s=0.69 \pm 0.067$ Vs. group B $45 s$ $=0.76 \pm 0.066$ Vs. $60 s=0.80 \pm 0.063 ; P<0.0001)$. Rotor density maps for group $B$ showed higher similarity and lower variation $(0.88 \pm 0.092)$ when compared to group $A$ $(0.53 \pm 0.134)$. Our results suggest that time duration $\leq$ $30 \mathrm{~s}$ is not sufficient to detect/track spatiotemporal organization of rotors in PersAF patients.
\end{abstract}

\section{Introduction}

Atrial fibrillation (AF) is the most common cardiac arrhythmia seen in clinical practice. It affects more than 30 million individuals worldwide and increases the risk of stroke fivefold. Although the incidence is higher in the elderly, the burden of AF is found over the entire adult population with associated morbidity, mortality and healthcare costs [1]. The mechanisms that initiate and sustain AF are not yet well characterized. Successful catheter ablation of persistent atrial fibrillation (PersAF) in clinical practice is still a significant challenge, and the role of rotational activity (rotors) around the atria in sustaining AF is still debated, with studies suggesting that wavelet re-entries are mainly responsible for its maintenance. Furthermore, there have been fundamental differences between the studies on rotors in reporting their prevalence and spatiotemporal stability [2]. In contrast, in a clinical study (focal impulse and rotor modulation-FIRM) Narayan et al. [3], used a 64electrode basket catheter (FIRMap ${ }^{\mathrm{TM}}$, Topera) with customized signal processing technique for detection of rotors and focal sources in human persAF and reported that rotors were present in approximately $90 \%$ of patients. Their results produced conflicting outcomes, however, due to the spatiotemporal complexities of the rotors and technical challenges in analyzing the intracardiac signals including fractionation, varying cycle lengths and complexities of activation patterns [4-5].

The main aim of this study is to use a phase mapping approach, to track the spatiotemporal stability of the rotors in persAF patients and provide further understanding of the length of time to record VEGMs signals needed to locate rotors during PersAF in human.

\section{Materials and Methods}

\subsection{The Characterization of Enrolled Patients}

This study recruited symptomatic PersAF patients $(\mathrm{N}=$ 10 Median age $=57.8$ years, Min 36.1, Max76.4), who underwent left atrial (LA) catheter ablation for the first time guided by three-dimensional (3D) Non-contact mapping (NCM) using a multi-electrode array catheter (MEA) (EnSite Velocity, St Jude Medical). Ethics approval to conduct the study was obtained from the local ethics committee and all procedures were performed with informed consent. 


\section{Electrophysiology Study}

Prior to the electrophysiology (EP) study all antiarrhythmic drugs aside from amiodarone were halted. During the procedure, a quadripolar catheter and steerable decapolar catheter were advanced through the femoral vein and guided, until positioned in the coronary sinus (CS) and His position in the right atrium (RA) respectively. For all patients under fluoroscopic guidance, the single trans-septal puncture technique was used to access the LA, and then both a conventional deflectable mapping catheter and a high-density NCM MEA catheter were deployed in the LA. All patients were given heparin to maintain the activated clotting time $\geq 300 \mathrm{~s}$. The 3D geometry of LA was reconstructed in real-time with catheter mapping (EnSite Velocity, St. Jude Medical), LA anatomical landmarks were annotated including Pulmonary Veins (PV), left atrial appendage (LAA), atrial roof, septum, anterior, posterior wall (PW), and mitral valve (MV). 2048 VEGMs with sampling frequency of $2034.5 \mathrm{~Hz}$ were simultaneously collected for 5 minutes, in addition to the 12-lead ECG. The data was transferred to a laptop and analysed using a research tool USURP-GUI developed by our research group (details in [5]). The area of interest High Dominant Frequency (HDF), was located and ablated [6], following this another 5 mins post-ablation data were recorded continuously for each patient, and then the MEA was removed and AF ablation using standard pulmonary vein isolation (PVI) procedure was performed.

\subsection{Data Processing}

The 5 min recording (2048 VEGMs with their associated 3D coordinates, and 12-lead ECG) were analysed offline using MATLAB (R2018a, MathWorks, USA). Signals were originally sampled at $2034.5 \mathrm{~Hz}$ and band-pass filtered $(1-150 \mathrm{~Hz})$ and then resampled at $512 \mathrm{~Hz}$ to reduce processing time. The surface ECG was band-pass filtered between $(0.5-50 \mathrm{~Hz})$. QRST subtraction was performed on the VEGMs to remove the far-field ventricular influence using the method developed by our group [7].

\subsection{Phase Analysis and Rotor Detection}

There is a sequence of processing steps to be followed to convert VEGMs into phase maps. This study used the phase mapping approach reported by Kuklik et al. [8], The phase data can be extracted using Hilbert transform (eqn.1)[9], with the phase defined as the angle between the analytic signal and original signal, while the phase extracted using Kuklik et al uses the 'sinusoidal recomposition method', where the signal is represented as a sum of sinusoidal wavelets with amplitude proportional to the negative slope of the unipolar VEGMs (eqn.2).

$$
\mathrm{H}(\mathcal{U})(t)=\frac{1}{\pi} P \int_{-\infty}^{\infty} \frac{u(t)}{t-\tau} d \tau
$$

Here, applying Hilbert transform into function $\mathrm{H}(U)(t)$, where $\mathrm{P}$ is the Cauchy principal value of the integral, to allowing calculation of instantaneous phase as follows:

$$
\varphi(t)=\arctan \left(\frac{-u(t)-u^{*}}{H(u)-u^{*}}\right)
$$

The method can be summarised in the following steps: (1) Recomposing the VEGMs from sinusoidal wavelets with amplitudes proportional to the negative slope of the electrogram using sinusoidal recomposition method, (2) applying the Hilbert transform on the recomposed sinusoids signals, followed by (3) calculating the instantaneous phase of each signal producing a phase map. Figure 2 (B) illustrates these different steps.

The PSs were automatically identified using an algorithm developed by our group based on topological charge method as described by Bray et al. 2001 [10]. The PS locations were determined where the phase progresses through a complete cycle from $(-\pi$ to $+\pi)$. The stable PSs are those tracked over time subject to threshold for distance and for time. In each time frame, the location of each PS was compared with its location in the previous frame, only PSs lasting over $100 \mathrm{~ms}$ were considered [1112]. A rotor was defined as a stable PS, which persists for at least $100 \mathrm{~ms}$ with a spatial threshold of a 5-node distance between consecutive frames.

Rotor density maps were generated in $2 \mathrm{D}$ and $3 \mathrm{D}$, in order to assess the spatiotemporal stability of the rotors. The VEGMs were divided into a total of 60 nonoverlapping segments of different time durations starting from $5 \mathrm{~s}, 10 \mathrm{~s}, 15 \mathrm{~s}$ and so on, until the whole $300 \mathrm{~s}$ VEGM recording was covered. The results were represented into, group A $\leq 30 \mathrm{~s}$ and, group B $>30 \mathrm{~s}$ to investigate the minimum time duration required to track sustained rotors. Density maps of different time durations were compared with the full $300 \mathrm{~s}$ recording (figure $3 \mathrm{~A}$ ), and Pearson's correlation (CORR) was used, in order to assess the similarity of the shorter time duration rotor density maps and the $300 \mathrm{~s}$ one.

\subsection{Statistical Analysis}

The statistical data were analyzed using Graphpad Prism (version 7.04 for Windows). The continuous variables with normal distribution were expressed as mean ( \pm standard deviation). Wilcoxon matched-pairs signed-rank test was used to analysing nonparametric paired multiple data, while non-parametric unpaired data were analysed with Mann-Whitney test. A value of $\mathrm{P}<$ 0.05 was considered significant.

\section{Results and Discussions}

Figure1 summarizes the histogram of the rotors' lifespan in the LA over the $300 \mathrm{~s}$ of VEGM recording for 
10 patients, Overall, as the time duration increased the number rotors detected gradually increased $(5 \mathrm{~s}=17.2 \pm$ 8.8 Vs. $300 \mathrm{~s}=998.3 \pm 436.5, \mathrm{P}<0.05)$. This was observed in all patients, thereby, stating the dominance of rotors in sustaining this complex arrhythmia.

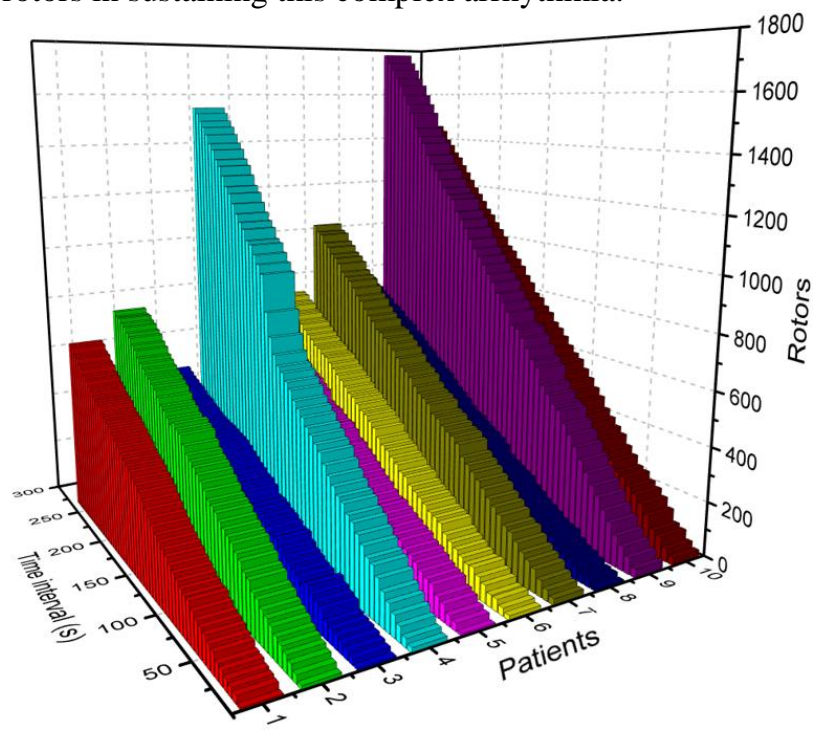

Figure 1: Histogram of rotors lifespan in the (LA) for 10 patients.

Whatever, electrophysiology mapping system is used to mapping $\mathrm{AF}$, there is a debate in the literature with regards to the required time duration of VEGMs to be used detecting/tracking rotors. Several studies have used

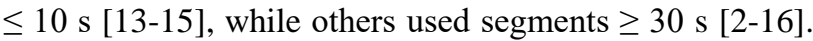
Thus, our results are expressed in two groups; group $\mathrm{A} \leq$ $30 \mathrm{~s}$ and group B > $30 \mathrm{~s}$. In order to investigate the time duration needed to produce a representative rotor density map, two time segments in each group were selected (group A $10-30 \mathrm{~s}$, group B 45-60 s) to distinguish the difference of the spatiotemporal changes of rotors in each rotor density map in comparison with the rotor density maps of $300 \mathrm{~s}$ segment recording (gold standard).

The results for all patients showed that the rotor density maps of group A had lower correlation (mean \pm SD: $10 \mathrm{~s}=0.47 \pm 0.064$. vs $30 \mathrm{~s}=0.69 \pm 0.067$, $P<0.0001)$, when compared with the gold standard, while for group B had higher correlation (mean \pm SD: $45 \mathrm{~s}=$ $0.76 \pm 0.066$, vs $60 \mathrm{~s}=0.80 \pm 0.063, P<0.0001)$ in comparison with the density map using the $300 \mathrm{~s}-$ long segment.

Figure 2 shows the rotor density maps in 3D for one patient. From the figure, it can be noticed that the 3D maps produced using the time duration (10 s up to $30 \mathrm{~s})$ (left maps) do not represent the actual spatiotemporal behaviour of rotors when compared with the gold standard rotor density map (Centrale map). Consequently, this may lead to misinterpretation of rotors' locations and targeting of false rotors. Therefore, time duration of $30 \mathrm{~s}$ or less are not adequate for producing maps representing the dynamics of rotors. In group B (right-hand side figure $2 \mathrm{~A}$ ) the location of the regions that host sustained rotors seemed to be consistent over the time duration and correlate better with the full $300 \mathrm{~s}$ map (centre).

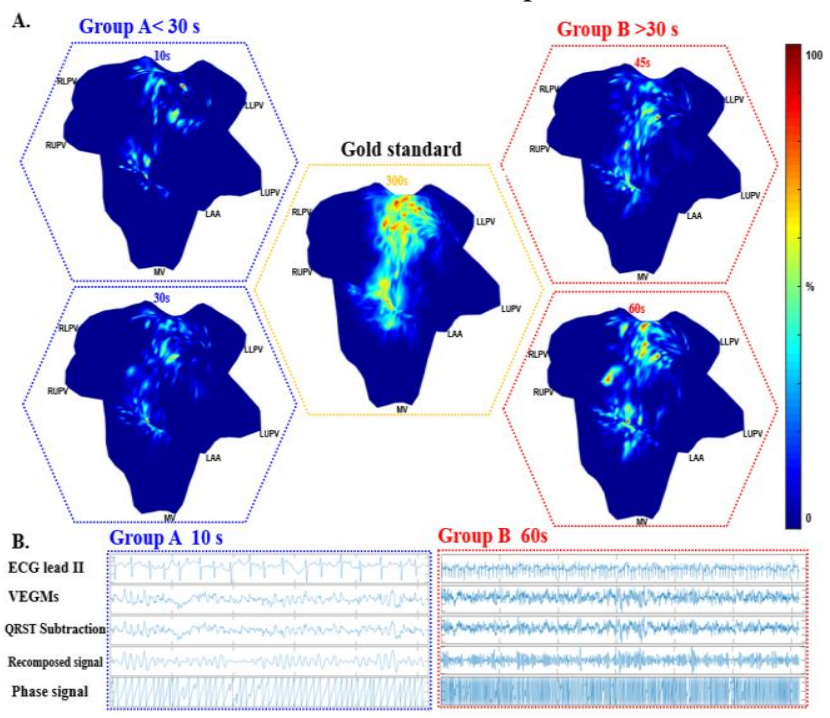

Figure 2(A), a comparison of rotor density maps for group A (10 s, $30 \mathrm{~s})$ and group B (45 s, $60 \mathrm{~s})$ against the full-length density map $300 \mathrm{~s}$ for one patient. The similarity results for group A were (CORR: $45 \%$, 64\%) Vs. group B (CORR: 76\%, 80\%). The colour bar indicates the region hosting sustained rotors for each density map. (B), an example of phase reconstruction of VEGMs of group A (10 s segment), and group B (60 s segment): starting with ECG lead II recording, original VEGMs recording followed by its subtracted version (after QRST subtraction) and finally, its recomposed signal (sum of sinusoidal wavelets) from which the corresponding instantaneous phase signal was calculated.

From the results in figure 3 (A), for all patients using the similarity index CORR. the rotor density maps of group A showing the similarity of $45-64 \%$ respectively, when compared to $300 \mathrm{~s}$ recording. Thus time duration $\leq 30 \mathrm{~s}$ is not sufficient to characterize the spatiotemporal behaviour of rotors and identifying the atrial regions that host the majority of rotors activities. The maps obtained from $60 \mathrm{~s}$ segment were much better correlated to the map using $300 \mathrm{~s}$ recording with similarity of $80 \%$. Figure 3 (B) shows a decreasing trend in the standard deviation for all patients, with $\mathrm{P}<0.0001$ presenting significant difference between the groups. Therefore $60 \mathrm{~s}$ is adequate to locate rotors.
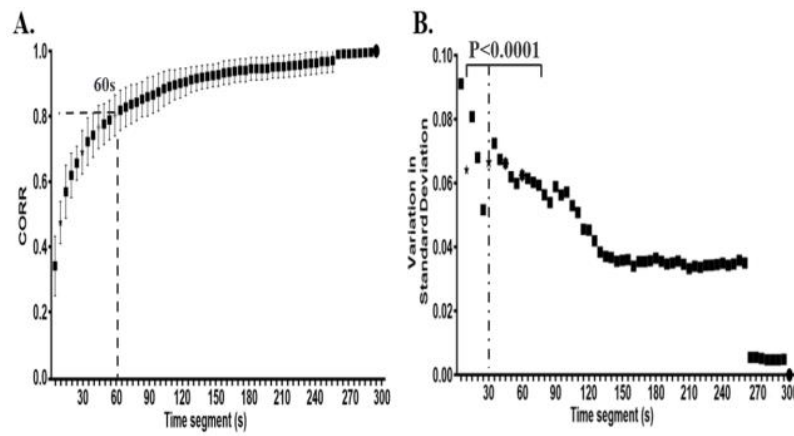

Figure 3: (A), Correlation coefficients CORR for 10 patients within the $60 \mathrm{~s}$ segments of VEGMs, (B) illustrates the drop standard deviation for 10 patients over the whole recorded segments as the length of data used increases. 


\section{Conclusions}

Tracking the stable drivers (rotors) can provide a clear picture of the characteristics of complex arrhythmias during AF. However, rotor-based ablation for persAF remains an ongoing debate, due to the lack of stability of spatiotemporal rotors' behaviour. Thus, the time duration of the VEGMs used for phase mapping has a significant effect on the identification of rotors. Our results suggest that VEGMs' duration $\leq 30 \mathrm{~s}$ is not long enough to characterise rotors in PersAF patients, and time duration of $60 \mathrm{~s}$ is our recommendation for identifying them.

\section{Acknowledgments}

The work was supported by the National Institute for Health Research (NIHR) Leicester Cardiovascular Biomedical Research Centre. XL received funding from the British Heart Foundation (BHF Project Grant no. PG/18/33/33780).

\section{References}

[1] Sheikh A, Patel NJ, Nalluri N, Agnihotri K, Spagnola J, Patel A, et al. "Trends In Hospitalization For Atrial Fibrillation: Epidemiology, Cost, And Implications For The Future," Progress in cardiovascular diseases. 2015;58(2):105-16.

[2] Sakata K, Okuyama Y, Ozawa T, Haraguchi R, Nakazawa $\mathrm{K}$, Tsuchiya T, et al. "Not All Rotors, Effective Ablation Targets For Nonparoxysmal Atrial Fibrillation, Are Included In Areas Suggested By Conventional Indirect Indicators Of Atrial Fibrillation Drivers: Ex TR A Mapping Project," Journal of arrhythmia. 2018;34(2):176-84.

[3] Narayan SM, Krummen DE, Shivkumar K, Clopton P, Rappel W-J, Miller JM. "Treatment of atrial fibrillation by the ablation of localized sources: CONFIRM (Conventional Ablation for Atrial Fibrillation With or Without Focal Impulse and Rotor Modulation) Trial," Journal of the American College of Cardiology. 2012;60(7):628-36.

[4] Chu G, Almeida TP, Chin SH, Varanasi S, Siddiqui S, Man S, et al. "29 Characterising The Relationship Between Force And Catheter Stability During Manual And Robotic Paroxysmal AF Ablation," Europace. 2014;16(suppl_3):iii12-iii.

[5] Li X, Salinet JL, Almeida TP, Vanheusden FJ, Chu GS, Ng GA, Schlindwein FS. "An Interactive Platform To Guide Catheter Ablation In Human Persistent Atrial Fibrillation Using Dominant Frequency, Organization And Phase Mapping," Computer methods and programs in biomedicine. 2017;141:8392.

[6] Salinet JL, Tuan JH, Sandilands AJ, Stafford PJ, Schlindwein FS, Ng GA. "Distinctive Patterns Of Dominant Frequency Trajectory Behavior In Drug-Refractory Persistent Atrial Fibrillation: Preliminary Characterization Of Spatiotemporal Instability," Journal of cardiovascular electrophysiology. 2014;25(4):371-9.

[7] Salinet JL, Madeiro JP, Cortez PC, Stafford PJ, Ng GA, Schlindwein FS. "Analysis of QRS-T Subtraction In Unipolar Atrial Fibrillation Electrograms," Medical \& biological engineering \& computing. 2013;51(12):1381-91.

[8] Kuklik P, Zeemering S, Maesen B, Maessen J, Crijns HJ, Verheule S, et al. "Reconstruction Of Instantaneous Phase Of Unipolar Atrial Contact Electrogram Using A Concept Of Sinusoidal Recomposition And Hilbert Transform," IEEE transactions on biomedical engineering. 2014;62(1):296-302.
[9] Umapathy K, Nair K, Masse S, Krishnan S, Rogers J, Nash $\mathrm{MP}$, et al. "Phase Mapping Of Cardiac Fibrillation," Circulation: Arrhythmia and Electrophysiology. 2010;3(1):10514.

[10] Bray MA, Lin SF, Aliev RR, Roth BJ, Wikswo Jr JP. "Experimental And Theoretical Analysis Of Phase Singularity Dynamics In Cardiac Tissue," Journal of cardiovascular electrophysiology. 2001;12(6):716-22.

[11] Salinet J, Schlindwein FS, Stafford P, Almeida TP, Li X, Vanheusden FJ, et al. "Propagation Of Meandering Rotors Surrounded By Areas Of High Dominant Frequency In Persistent Atrial Fibrillation," Heart Rhythm. 2017;14(9):126978.

[12] Samie FH, Berenfeld O, Anumonwo J, Mironov SF, Udassi $\mathrm{S}$, Beaumont $\mathrm{J}$, et al. "Rectification Of The Background Potassium Current: A Determinant Of Rotor Dynamics In Ventricular Fibrillation," circul Research. 2001;89(12):1216-23. [13] Zaman JA, Sauer WH, Alhusseini MI, Baykaner T, Borne RT, Kowalewski CA, et al. "Identification And Characterization Of Sites Where Persistent Atrial Fibrillation Is Terminated By Localized Ablation", Circulation: Arrhythmia and Electrophysiology. 2018;11(1):e005258.

[14] Aronis KN, Ashikaga H. "Impact Of Number Of CoExisting Rotors And Inter-Electrode Distance On Accuracy Of Rotor Localization," Journal of electrocardiology. 2018;51(1):82-91.

[15] Kuklik P, Zeemering S, van Hunnik A, Maesen B, Pison L, Lau DH, et al. "Identification Of Rotors During Human Atrial Fibrillation Using Contact Mapping And Phase Singularity Detection: Technical Considerations," IEEE Transactions on Biomedical Engineering. 2016;64(2):310-8.

[16] Houben RP, Allessie MA. "Processing Of Intracardiac Electrograms In Atrial Fibrillation, "IEEE Engineering in Medicine and Biology Magazine. 2006;25(6):40-51.

Address for correspondence:

Mahmoud Ehnesh

University Rd, Leicester LE1 7RH me196@le.ac.uk 